daily, with total loss of appetite, marked her wretched existence till the 29th of March, when death closed the scene ; she having taken little or no nourishment the last thirty or forty days of her existence.

Post-mortem Examination 36 hours after Death.-On opening the abdomen, the general appearance of congestion was evident. The lower flexure of the colon adhered to the bladder, and these to t'ie fundus uteri, so firmly that they could not be separated without the scalpel ; in doing which, an abscess was opened between the right kidney and the crest of the ileum. On raising the pelvic viscera, they were so completely changed by disease that it was difficult to distinguish the organs one from another. From the os externum, through the vagina, was one mass of fungus, of a fleshy, firm appearance. The os tince and cervix uteri seemed to have sloughed off, and their place to be occupied by the excrescence ; so that the fundus uteri was the only part of the pelvic viscera that retained the natural appearance, except the rectum. 'The left ovarium was enlarged and indurated. The rigltt seemed to be occupied by the abscess. The left kidney was three times as large as the riglth, and somewhat firm in its texture. The liver was enlarged to the size of a two quart bottle, and much indurated ; gallbladder partly filled with very darkcolored bile; - thoracic viscera pretty natural.

Lisbon, $\mathcal{N}$. $\boldsymbol{H}$., Mlarch, 1833. Moses Hibard, M.D.

\title{
CASE OF SPINAL IRRITATION.
}

[Communicated for the Boston Medical and Surgical Journal.]

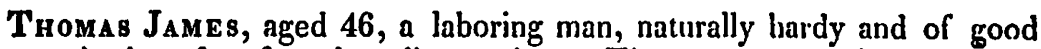
constitution, free from hereditary taint. Five years ago began to be troubled with morbid symptoms of the chest, with pain in the side, disturbed rest, and general debility. These difficulties continued to increase each sucreeding year-being occasionally palliated by remedial measures, and again recurring with redoubled force.

In October, 1832, he had a fresh attack of his complaints, attended with a high degree of feverish excitement and great nervous irritability. After the vascular excitement 'wore off,' he was kept in a state of extreme debility and great derangement of the nervous functions. In this state I first saw him, December 18th. His countenance was pallid, moderately emaciated; the pneumonic symptoms inuch aggravated; no location of the disease in any one part of the lungs, but a general feeling of 'fullness' and great oppression throughout the chest, and difficult respiration ; rest very much disturbed; cough slight ; expectoration very triffing; pain in the right side constant and distressing, occasionally very severe and lancinating. Digestive functions healthy and natural, except some slight dyspeptic symptoms. As to courage and animal spirits, he was entirely destitute of them - being dejected, discouraged, and despairing of ever recovering his health. His physician had abandoned him to his fate-having exlinusted his professional resources, and virtually acknowledged himself in the dark. His family connections and neighbor's had it settled in their minds that he must soon die. 
On making pressure up and down the spine, the sixth and seventh dorsal vertebræ were found exquisitely tender. Recourse was then had to thorough cupping and scarifications in the neighborhood of the diseased part, followed with an extensive vesication directly over the spine. An immediate amendment was the result. The cupping, \&c. with the consequent blister, were repeated once in eight days. In four weeks from the commencement, it was thought advisable to substitute setons for the cupping, \&c. Accordingly, one was introduced near the diseased vertebræ ; another at the origin of the third portion of the serratus magnus of the right side-and the man left to take care of himself.

The convalescence in this case has been steady and uniform, from the commencement of the above treatment to the present time. Then he was entirely confined to his room, and generally to his bed; now he is able to attend to his domestic affairs without inconvenience, except the slight soreness from the setons, which he chooses yet to retain.

In this case no medicines were used internally, save a slightly tonic preparation-reliance being placed almost entirely upon the external 'back-bone' treatment. A nourishing diet was enjoined, and practised during the whole course.

Leeds, Me., February 14, 1833.

\section{HEMORRHAGE FROM THE UNIMPREGNATED UTERUS.}

To the Editor of the Bosion.Medical and Surgical Journal.

SIR,-I remarked, in a communication in your last, that the tampon was stated to be only applicable to certain cases of uterine hemorrhage, and among these was mentioned hemorrhage from the unimpregnated uterus. I have always supposed that hemorrhage never occurred under such circumstances, although I do not recollect to have seen the fact stated in any medical work. The question is certainly a very interesting one, and not wanting in importance. Indeed, the character, and therefore the happiness of many' an innocent female is liable to be destroyed, if the idea I lave always entertained on this subject be incorrect. It is to be hoped, therefore, that you, or some of your readers who are better acquainted with the subject than myself, will afford us some light on it, through the medium of your pages.

Boston, March 4, 1833.

Respectfully yours, Medicus, Jr.

[We shall be happy to publish any communications from the faculty on this important subject.-Ev.]

\section{CASE OF PROTRACIED VOMITING.}

Extraordinary Case of Protracted Vomiting, in which Life was sustained for an unusual length of time without Food. By Daniel Sexton, M.D., of New Harmony.

I was requested on the 17 th of October, 1829 , to visit Mrs. L. L., who had been for some time declining in health. For five weeks she had been confined to her bed, vomiting frequently, and unable to 